\title{
IMPACTO DE LOS CULTIVOS SOBRE LOS EFECTOS DE LAS INUNDACIONES. VALORCIÓN ECONÓMICA DEL SERVICIO DE MITIGACIÓN DEL RIESGO.
}

\author{
$\underline{\text { Víctor Martínez }}^{a}{ }^{*}$, José Antonio Albaladejo $^{b}$, José Ángel Zabala $^{a}$ \\ ${ }^{a}$ Universidad Politécnica de Cartagena (Cartagena, victor.martinez@upct.es), ${ }^{\boldsymbol{b}}$ Universidad de Murcia
}

\begin{abstract}
Resumen
Los agroecosistemas, además del servicio de provisión de alimentos, proporcionan otros Servicios Ecosistémicos (SE), entre los cuales se encuentra el de Mitigación del Riesgo de Inundación (SEMRI) en la población. Este servicio de regulación ha sido escasamente estudiado en la literatura científica, que ha atendido principalmente a factores clave como los patrones de usos del suelo e indicadores intermedios, ignorando aspectos biofísicos clave que condicionan el desarrollo de una inundación y su impacto sobre la sociedad. En este contexto, el objetivo de este trabajo es valorar económicamente el SEMRI que pueden proveer los agroecosistemas. Para ello, se propone emplear el método de daños evitados, realizando una modelización hidrológica e hidráulica bidimensional que traslade en el espacio y tiempo las escorrentías generadas, a partir de la cual se puede estimar el valor del daño en las viviendas mediante una función de pérdidas. El modelo así construido permite a su vez cuantificar la reducción de daños asociados a episodios de inundación en distintos escenarios de ocupación de cultivos maximizadores del SEMRI. El área de estudio elegida es el municipio de Los Alcázares, localizado en el Campo de Cartagena (Murcia), población afectada de forma recurrente por inundaciones tras episodios de lluvias torrenciales.
\end{abstract}

Palabras clave: Agroecosistemas, Servicios Ecosistémicos, Servicios de Regulación, Inundaciones

\section{Introducción y objetivos}

Las inundaciones son el riesgo natural más frecuente y al cual se encuentra expuesto una mayor parte de la población [UNISDR y CRED (2015)]. Esto unido al aumento previsto en la frecuencia e intensidad de las precipitaciones extremas [IPCC (2014)] hace que las medidas de defensa contra inundaciones adquieran aún más relevancia, siendo especialmente relevantes las de tipo no estructural, basadas en los propios ecosistemas [Barth y Döll (2016)].

Los ecosistemas proporcionan contribuciones al bienestar humano que son conocidas como Servicios Ecosistémicos (SE) [Haines-Young y Potschin (2018)], y son generalmente clasificados en servicios de provisión, culturales y de regulación [MEA (2005)]. Entre estos últimos, se encuentra el Servicio Ecosistémico de Mitigación del Riesgo de Inundación (SEMRI) [Van der Ploeg y de Groot (2010)], el cual se reconoce como la capacidad de retención del agua de la vegetación y el suelo durante una inundación, lo cual contribuye al bienestar humano reduciendo las escorrentías, picos de crecida y daños asociados al suceso [MEA (2005)].

El estudio del SEMRI ha sido escaso en la literatura [Qiu (2019)]. Las metodologías empleadas han asumido relaciones directas entre indicadores intermedios y el potencial de regulación [Smithers et al. (2016)] o empleado modelos hidráulicos unidimensionales, poco adecuados en llanuras o espacios urbanos [Lawrence et al. (2019)]. Las limitaciones no solo son metodológicas, ya que únicamente se ha estudiado la provisión de este servicio por parte de ecosistemas naturales.

Los agroecosistemas son ecosistemas antropizados que además de los servicios de provisión de alimentos, pueden proveer de otros SE, entre los cuales se encuentra el SEMRI [Power (2010)]. Esta provisión de SE depende de la actividad humana que se realiza en ellos mediante prácticas agrícolas. Dado que la gestión de los agroecosistemas puede resultar de especial relevancia para la mitigación del riesgo de inundación, estos deben ser tenidos en cuenta en la formulación de medidas de defensa y mitigación de daños contra estos episodios.

En este contexto, el objetivo de este trabajo es valorar económicamente el SEMRI que proveen los agroecosistemas. Para ello, se emplea el método de daños evitados, lo cual implica realizar una modelización hidrológica e hidráulica bidimensional que traslade en el espacio y tiempo las escorrentías generadas, a partir de lo cual puede estimarse el daño económico en las viviendas a través de una función de pérdidas. El caso de estudio seleccionado es el municipio de los Alcázares, un ejemplo de núcleo urbano ubicado en un territorio predominantemente agrícola y que es afectado recurrentemente por inundaciones.

\section{Metodología}

El municipio de Los Alcázares (Murcia) se ubica en la desembocadura de las ramblas del Albujón y de la Maraña (Mapa 1), dos de los principales cauces de la cuenca de drenaje del Campo de Cartagena. Este 
espacio se caracteriza por una alta variabilidad inter e intra-anual en sus precipitaciones, habiéndose registrado episodios de gran intensidad que igualan los aproximados 300mm de precipitación media anual en 12 horas. El territorio se ha visto intensamente modificado durante las últimas décadas, habiéndose desarrollado una agricultura de regadío intensiva que actualmente ocupa un 72,46\% de la superficie, de la cual el 44,05\% son cultivos herbáceos de regadío, el 12,7\% cítricos y el 1,07\% invernaderos, mientras que los leñosos de secano suponen un 14,64\% del área. Además, los drenajes de las ramblas mencionadas se ven interrumpidos antes de llegar al núcleo urbano por una autopista sobre-elevada, lo cual altera considerablemente su flujo.

Mapa 1. Área de estudio

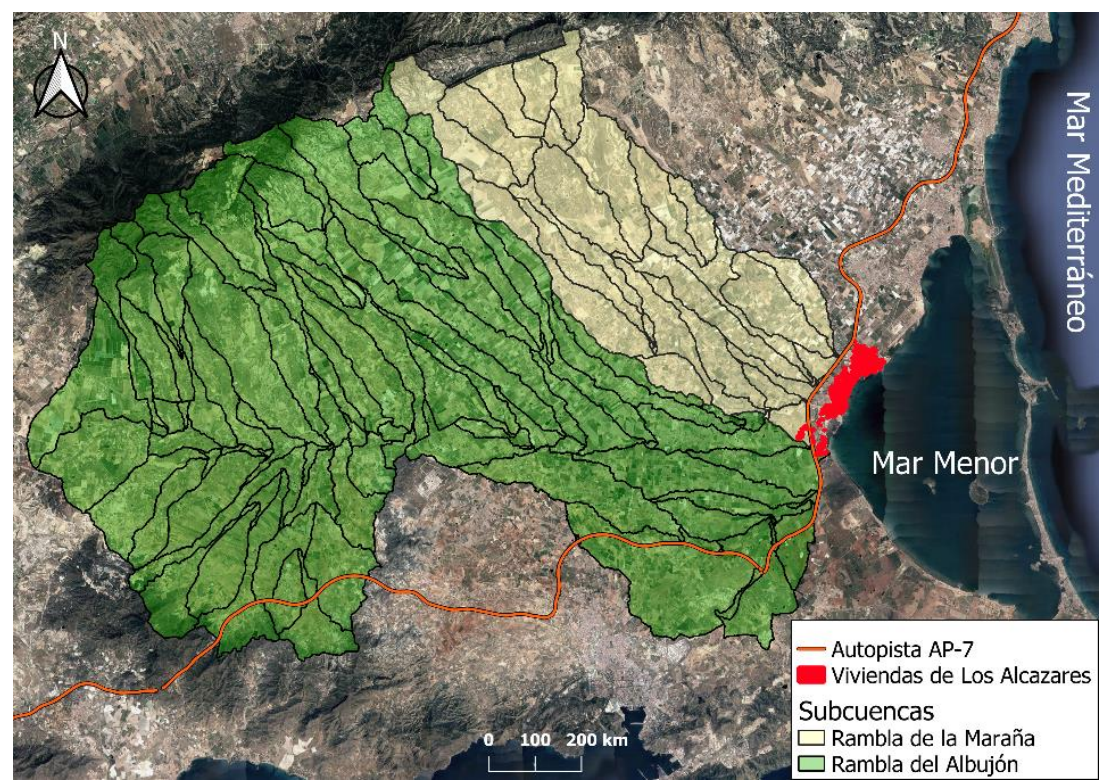

El método empleado para la valoración económica ha sido el de daños evitados. Para su aplicación se ha realizado una modelización hidrológica e hidráulica mediante las cuales se trasladan, en el espacio y tiempo, las escorrentías generadas por las precipitaciones correspondientes a los Periodos de Retorno (PR) 10, 100 y 500 años, siendo el inverso asociado a estos PR la probabilidad anual de ocurrencia del fenómeno. La cartografía de la inundación obtenida de estos modelos es empleada para estimar los daños económicos en las viviendas a través de una función de pérdidas. El flujo de acciones y datos necesarios para la aplicación del método de daños evitados viene recogido en el Gráfico 1.

Gráfico 1. Diagrama de procesos de la metodología

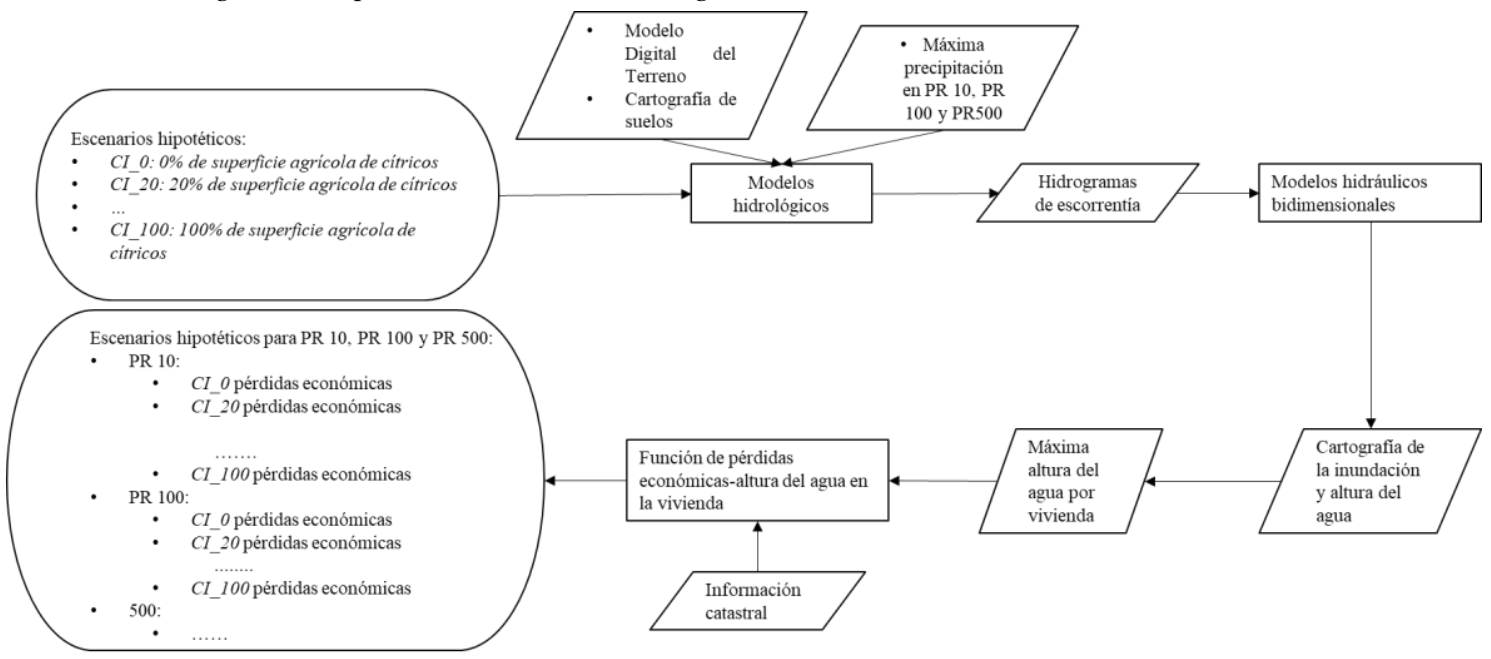

La cuantificación de daños se ha realizado en 5 escenarios hipotéticos donde se incrementa la extensión de los cultivos identificados como maximizadores del SEMRI por su elevado umbral de escorrentía (RT). Según el Sistema Nacional de Cartografía de Zonas Inundables (SNCZI), dentro de los cultivos presentes en la zona, estos serían los cítricos [MARN (2011)]. Dichos escenarios parten de la situación actual, con un $12,7 \%$ de la superficie dedicada a cítricos, con incrementos sucesivos hasta el $100 \%$ de la superficie 
agrícola. De esta forma, se obtienen los daños asociados a distintos valores de RT medio en las cuencas hidrográficas de las ramblas estudiadas.

La cartografía de usos del suelo se ha obtenido combinando el Sistema de Información Geográfica de Parcelas Agrícolas (SIGPAC) para los usos agrícolas y Corine Land Cover 2018 (CLC) para el resto de usos del suelo. Los modelos hidrológicos se han realizado mediante el software HEC-HMS aplicando el método del hidrograma unitario adimensional, mientras que para los modelos hidráulicos se ha empleado HEC-RAS y su módulo de cálculo bidimensional, aplicando la ecuación "Full Momentum” (Saint-Venant). Estos han requerido inputs adicionales, como un Modelo Digital del Terreno (MDT) obtenido del Centro de Descargas del Instituto Geográfico Nacional (CNIG), la cartografía de suelos suministrara por el Instituto Geográfico y Minero (IGME) y la máxima precipitación en 24 horas para los PR del Mapa de Caudales Máximos en Régimen Natural (MCMRN). La función de pérdidas y la fórmula empleada para calcular el Daño Anual Medio (DAM) por vivienda han sido las propuestas por Manrique et al. (2017).

$$
\mathrm{D}=\sum_{\mathrm{i}=1}^{\infty} \frac{\mathrm{D}\left(\mathrm{x}_{\mathrm{i}-1}\right)+\mathrm{D}\left(\mathrm{x}_{\mathrm{i}}\right)}{2}\left[\mathrm{P}\left(\mathrm{x} \geq \mathrm{x}_{\mathrm{i}-1}\right)-\mathrm{P}\left(\mathrm{x} \geq \mathrm{x}_{\mathrm{i})}\right]\right.
$$

Donde $\mathrm{D}(\mathrm{x})$ es el daño que resultaría de un evento concreto $x$ y $\mathrm{P}(\mathrm{x})$ es la probabilidad anual del evento.

\section{Resultados}

Los resultados reflejan una reducción de las pérdidas económicas al aumentarse la superficie de cítricos en los distintos PR (Gráfico 2). La máxima reducción de pérdidas respecto a la situación actual es alcanzada en el escenario donde mayor superficie es ocupada por cítricos (100\% CI), siendo esta del 24,75\%, 18,49\% y 9,82\% para los PR 10, 100 y 500 respectivamente. Esto indica un límite en la regulación frente a los fenómenos más extremos.

Gráfico 2. Curva de pérdidas por Periodo de Retorno $(P R)$ y escenario

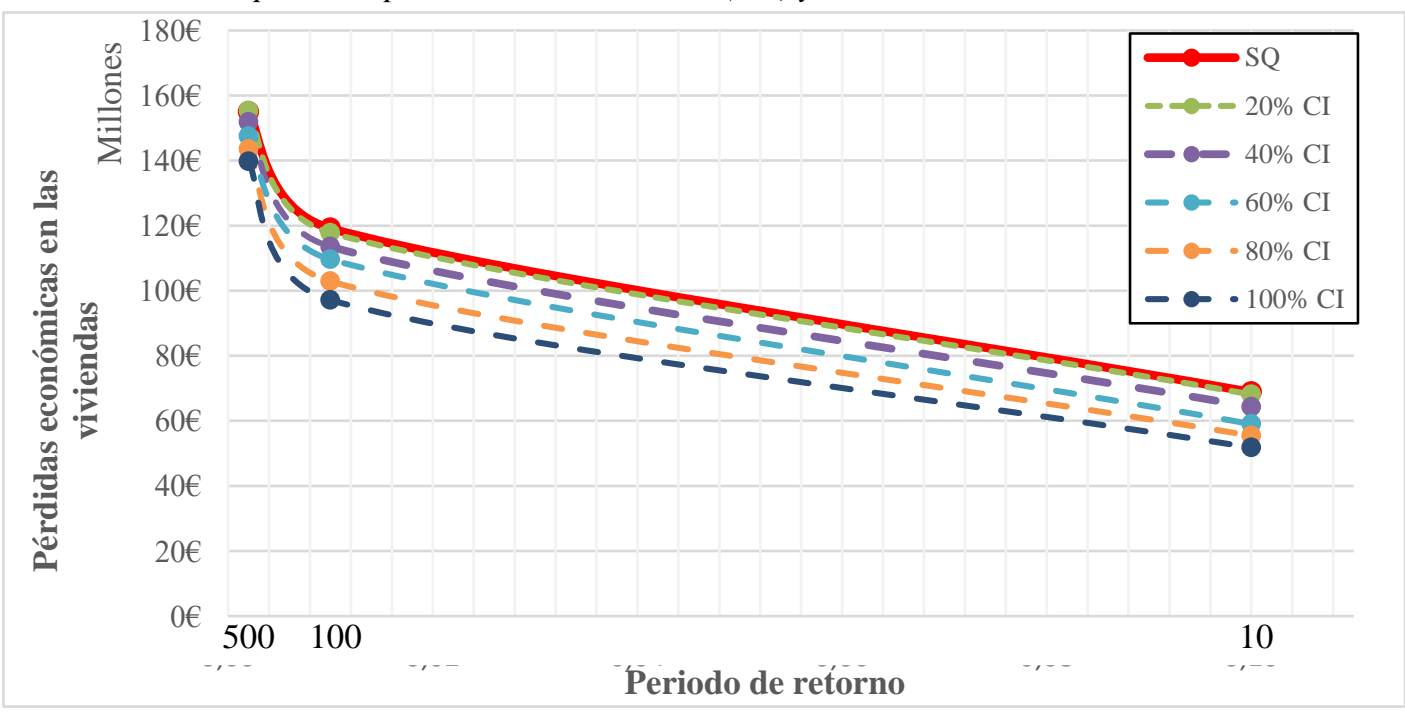

El máximo cambio que se da en el RT medio de las dos cuencas hidrográficas es de 6,98 unidades (Cuadro 1). La relación entre el RT y el DAM parece ser lineal, obteniéndose un valor de $451.391 €$ /año de beneficios por cada unidad reducida en el RT medio de las cuencas hidrográficas de las dos ramblas. Para el caso de los cítricos, esto supondría según sus valores de RT, un valor medio de 24,51€/ha/año.

Cuadro 1. Variación del Daño Anual Medio (DAM) por escenario

\begin{tabular}{ccccc}
\hline Escenario & $\begin{array}{c}\text { Umbral de } \\
\text { escorrentía medio }\end{array}$ & $\begin{array}{c}\text { Superficie cítricos (ha) } \\
\text { Actual }\end{array}$ & $\begin{array}{c}\text { Daño Anual Medio } \\
\text { (DAM) }\end{array}$ & $\Delta$ DAM (\%) \\
\hline $\mathbf{2 0 \%}$ CI & 20,61 & 14.294 & 9.556 .290 & - \\
$\mathbf{4 0 \%} \mathbf{C I}$ & 22,66 & 20.093 & 9.458 .887 & $-1,02 \%$ \\
$\mathbf{6 0 \%} \mathbf{C I}$ & 24,05 & 40.186 & 9.066 .309 & $-5,13 \%$ \\
$\mathbf{8 0 \%} \mathbf{C I}$ & 25,8 & 60.279 & 8.619 .111 & $-9,81 \%$ \\
$\mathbf{1 0 0 \%} \mathbf{C I}$ & 27,59 & 80.372 & 8.110 .360 & $-15,13 \%$ \\
\hline
\end{tabular}




\section{Conclusiones}

La valoración del SEMRI de los agroecosistemas adquiere relevancia ante la necesidad de evaluar medidas de defensa contra las inundaciones no estructurales basadas en la naturaleza. La metodología propuesta permite valorar este servicio de forma precisa en distintos contextos como entornos urbanos o llanuras de inundación, así como en distintos escenarios de cambio de usos del suelo agrícolas.

Sin embargo, esta metodología tiene límites en su aplicación, ya que requiere gran cantidad de inputs y capacidad de procesamiento. Esto reduce su posibilidad de aplicación a casos concretos de pequeña extensión. Además, la estimación de daños realizada es conservadora, ya que solo se tienen en cuenta daños directos en las viviendas.

Desde la agricultura, para el área de estudio puede proveerse un SEMRI de $451.391 € /$ año por cada unidad reducida en el RT medio, lo cual supone para el caso de los cítricos, cultivo maximizador del servicio, un valor de $24,51 € / \mathrm{ha} / a n ̃ o$. Esta información es de gran utilidad apoyar la toma de decisión de los organismos encargados de la gestión territorial y del riesgo en esta área de estudio.

\section{Agradecimientos}

Este trabajo se ha realizado en el marco del proyecto 20912/PI/18 financiado por la "Fundación SénecaAgencia de Ciencia y Tecnología de la Región de Murcia". Víctor Martínez agradece la ayuda recibida del MEFP (FPU19/05143).

\section{Bibliografía}

Barth, N. C., \& Döll, P. (2016). Assessing the ecosystem service flood protection of a riparian forest by applying a cascade approach. Ecosystem Services, 21, 39-52. https://doi.org/10.1016/j.ecoser.2016.07.012

Haines-Young, R., \& Potschin, M. B. (2018). Common international classification of ecosystem services (CICES) V5. 1 and guidance on the application of the revised structure. Nottingham: Fabis Consulting Ltd.

IPCC. (2014). Climate Change 2014 Part A: Global and Sectoral Aspects. In Climate Change 2014: Impacts, Adaptation, and Vulnerability. Part A: Global and Sectoral Aspects. Contribution of Working Group II to the Fifth Assessment Report of the Intergovernmental Panel on Climate Change. papers2://publication/uuid/B8BF5043-C873-4AFD-97F9-A630782E590D

Lawrence, C. B., Pindilli, E. J., \& Hogan, D. M. (2019). Valuation of the flood attenuation ecosystem service in Difficult Run, VA, USA. Journal of Environmental Management, 231, 1056-1064. https://doi.org/10.1016/j.jenvman.2018.10.023

Manrique, D., Nájera, A., Escartín, C., Moreno, C., Martínez, E., Espejo, F., Sánchez, F. J., Aparicio, M., Cordero, S., \& Gónzalez, S. (2017). Guía para la Reducción de la Vulnerabilidad de los Edificios Frente a las Inundaciones (Consorcio de Compensación de Seguros (CCS) (ed.)).

MARN (Ministerio de Medio Ambiente Medio Rural y Medio Marino). (2011). Guía Metodológica para el desarrollo del Sistema Nacional de Cartografía de Zonas Inundables (Secretaría General Técnica del Ministerio de Medio Ambiente y Medio Rural y Marino (ed.)).

https://www.miteco.gob.es/es/agua/publicaciones/guia_metodologica_ZI.aspx

Power, A. G. (2010). Ecosystem services and agriculture: Tradeoffs and synergies. Philosophical Transactions of the Royal Society B: Biological Sciences, 365(1554), 2959-2971. https://doi.org/10.1098/rstb.2010.0143

Qiu, J. (2019). Effects of Landscape Pattern on Pollination, Pest Control, Water Quality, Flood Regulation, and Cultural Ecosystem Services: a Literature Review and Future Research Prospects. Current Landscape Ecology Reports, 4(4), 113-124. https://doi.org/10.1007/s40823-019-00045-5

Smithers, R., Korkeala, O., Whitely, G., Brace, S., \& Holmes. (2016). Valuing flood-regulation services for inclusion in the UK ecosystem accounts (Issue 2).

Van der Ploeg, S., \& de Groot, R. S. (2010). The TEEB Valuation Database-a searchable database of 1310 estimates of monetary values of ecosystem services. Foundation for Sustainable Development, Wageningen, The Netherlands. 